\title{
A STABILITY PROPERTY OF STOCHASTIC VIBRATION
}

\author{
M. ELSHAMY,* Alabama A\&M University
}

\begin{abstract}
Let $u(t, x)$ be the displacement at time $t$ of a point $x$ on a string; the time variable $t$ varies in the interval $I:=[0, T]$ and the space variable $x$ varies in the interval $J:=[0, L]$, where $T$ and $L$ are fixed positive constants. The displacement $u(t, x)$ is the solution to a stochastic wave equation. Two forms of random excitations are considered, a white noise in the initial condition and a nonlinear random forcing which involves the formal derivative of a Brownian sheet. In this article, we consider the continuity properties of solutions to this equation. Smoothness characteristics of these random fields, in terms of Hölder continuity, are also investigated.
\end{abstract}

Keywords: Stochastic wave equation; Brownian sheet; convergence

2000 Mathematics Subject Classification: Primary 60H15; 60H30

\section{Introduction}

Stochastic partial differential equations arise in the description of physical systems which are subject to random effects. Flexible structures, for example, are influenced by random disturbances due to wind loading conditions as well as other random forces. The study of motion due to such random effects gives rise to the analysis of vibrations which involve some form of random perturbations. The vibrations of a string excited by different types of randomness have been studied by several authors, for example Orsingher (1984), (1989), Cabaña (1972), (1991), Elshamy (1995), (1996), and Belinskiy and Caithamer (2001). In the study of deterministic systems, there are a number of possible stability concepts. For the stochastic case, there are many more. This is because the problem of stability is essentially a problem of convergence. For each stability definition in the deterministic case there are several corresponding definitions in the stochastic case, which are generated by the different modes of stochastic convergence. Here, brief examples of stochastic stability consistent with the mean square convergence, $L^{p}$-convergence, and convergence in probability for a stochastic wave equation are given. In this article we consider a nonlinear stochastic wave equation in which the space dimension is one. In this case, the stochastic wave equation admits solutions which are functions in the usual sense. We show that the solutions to the stochastic wave equation depend continuously on its coefficients. We consider two models of stochastic vibrations, one in the form of initial white noise and the other a nonlinear random forcing which involves the formal derivative of a Brownian sheet. The displacement $u(t, x)$ is governed by a nonlinear stochastic wave equation. The time variable $t$ varies in the interval $I:=[0, T]$ and the space variable $x$ varies in the interval $J:=[0, L]$, where $T$ and $L$ are fixed positive constants. A Dirichlet boundary condition is imposed for the solutions to the wave equation.

Received 10 July 2006; revision received 12 February 2007.

* Postal address: Department of Mathematics, Alabama A\&M University, Normal, AL 35762, USA. 
Since Gronwall's inequality will be used more than once, we state a version of it in the following result.

Lemma 1.1. Suppose that $g \geq 0$ and h are integrable on $\left[t_{0}, T\right]$ and

$$
g(t) \leq b \int_{t_{0}}^{t} g(s) \mathrm{d} s+h(t), \quad t_{0} \leq t \leq T, b>0 .
$$

Then

$$
g(t) \leq h(t)+b \int_{t_{0}}^{t} \mathrm{e}^{b(t-s)} h(s) \mathrm{d} s, \quad \text { for } t_{0} \leq t \leq T .
$$

\section{Initial white noise excitation}

Consider the vibration of a string of length $L$ tied at both ends. The vibration is triggered by an external force $f$ and a white noise disturbance in the initial condition. The displacement $u(t, x)$ of a point $x$ at time $t$ on the string is described by the following version of the stochastic wave equation:

$$
\begin{aligned}
\frac{\partial^{2} u(t, x)}{\partial t^{2}} & =\frac{\partial^{2} u(t, x)}{\partial x^{2}}+f(x, u), \\
u(0, x) & =a(x), \\
\left.\frac{\partial u(t, x)}{\partial t}\right|_{t=0} & =\dot{w}(x), \\
u(t, 0) & =\left.\frac{\partial u(t, x)}{\partial t}\right|_{x=L}=0, \quad(t, x) \in I \times J,
\end{aligned}
$$

where $\dot{w}(x)$ is a white noise. The initial condition $a(x)$ is a deterministic continuous function. The requirements of $f$ are continuity as a function of $(x, u)$ in $J \times \mathbb{R}$ and Lipschitz in $u$. The standard Brownian motion $w$ is defined on a fixed complete probability space $(\Omega, F, \mathrm{P})$ which supports all our random processes. The solution to (2.1) can be written as

$$
u(t, x)=u_{0}(t, x)+\frac{1}{2} \int_{I \times J} \int \chi_{(t, x)}(s, y) f(y, u(s, y)) \mathrm{d} y \mathrm{~d} s, \quad(t, x) \in I \times J,
$$

where

$$
\chi_{(t, x)}(s, y)=\sum_{k=-\infty}^{\infty}\left[\Psi_{(t, x+2 k L)}(s, y)-\Psi_{(t,-x+2 k L)}(s, y)\right]
$$

and

$$
\Psi_{(t, x)}(s, y)= \begin{cases}1, & \text { for } 0 \leq s \leq T, 0 \leq y \leq L, \text { and }|x-y| \leq t-s, \\ 0, & \text { otherwise. }\end{cases}
$$

The function $\chi_{(t, x)}$ vanishes except in the shaded region of Figure 1, where it takes successively the values +1 and -1 .

The random field $u_{0}(t, x)$ is given by

$$
u_{0}(t, x)=\frac{1}{2}[A(x+t)+A(x-t)]+\frac{1}{2} \int_{x-t}^{x+t} \bar{W}(\mathrm{~d} y),
$$




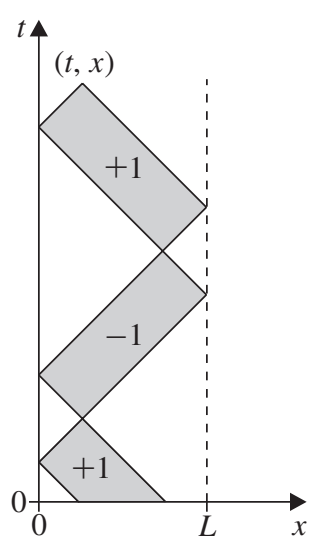

FIGURE 1: Graph of the function $\chi_{(t, x)}$.

where

$$
A(x)= \begin{cases}a(x), & \text { if } 0 \leq x \leq L, \\ -a(-x), & \text { if }-L \leq x \leq 0\end{cases}
$$

and

$$
\begin{aligned}
A(x+2 k L) & =A(x), & & \text { if }-L \leq x \leq L \text { and } k= \pm 1, \pm 2, \ldots, \\
\bar{W}(x) & :=w(x), & & \text { for } 0 \leq x \leq L, \\
\bar{W}(-x) & :=-w(x), & & \text { for } 0 \leq x \leq L, \\
\bar{W}(x+2 k L) & =\bar{W}(x), & & \text { if }-L \leq x \leq L \text { and } k= \pm 1, \pm 2, \ldots
\end{aligned}
$$

Equation (2.2) is identical to its deterministic counterpart, the initial conditions appear in $u_{0}(t, x)$, while the forcing term $f$ is the kernel of the third member. The velocity of the wave is assumed to be equal to 1. Particular cases of (2.2) have been examined in the literature. For example, Cabaña (1972), (1991) considered the case $a=w=0$ with $f=f(x, t)$. However, the problem considered in Cabaña (1991) is not in the framework of this article. Orsingher (1989) considered the case $a=0, f=0$, with the aim of studying the distribution of the maximum of the random field $u(t, x)$.

Let $f_{n}(x, u)$ be a sequence of continuous functions defined on $J \times \mathbb{R}$ which are Lipschitz in $u$. That is, a positive constant $k$ exists such that

$$
\left|f_{n}(x, u)-f_{n}(x, v)\right| \leq k|u-v| .
$$

Let $u_{n}(t, x)$ denote the solutions to the stochastic equation

$$
\begin{aligned}
\frac{\partial^{2} u_{n}(t, x)}{\partial t^{2}} & =\frac{\partial^{2} u_{n}(t, x)}{\partial x^{2}}+f_{n}\left(x, u_{n}\right), \\
\left.\frac{\partial u_{n}(t, x)}{\partial t}\right|_{t=0} & =\dot{w}(x), \\
u_{n}(t, 0) & =\left.\frac{\partial u_{n}(t, x)}{\partial t}\right|_{x=L}=0, \quad(t, x) \in I \times J .
\end{aligned}
$$


That is,

$$
u_{n}(t, x)=u_{0}(t, x)+\frac{1}{2} \int_{I \times J} \int \chi_{(t, x)}(s, y) f_{n}\left(y, u_{n}(s, y)\right) \mathrm{d} y \mathrm{~d} s, \quad(t, x) \in I \times J,
$$

where $u_{0}(t, x)$ is as given in (2.3).

The solutions to (2.5) depend continuously on the external force $f$ as well as the white noise $\dot{w}$ in the initial condition. We present this in the following result.

Lemma 2.1. Assume that $f_{n}(x, u) \rightarrow f(x, u)$ uniformly on $J \times \mathbb{R}$. Then we obtain

$$
\sup _{(t, x) \in I \times J} \mathrm{E}\left|u_{n}(t, x)-u(t, x)\right| \rightarrow 0 \quad \text { as } n \rightarrow \infty .
$$

Proof. Note that

$$
\begin{aligned}
u_{n}(t, x)-u(t, x)= & \frac{1}{2} \int_{I \times J} \int \chi_{(t, x)}(s, y) f_{n}\left(y, u_{n}(s, y)\right) \mathrm{d} y \mathrm{~d} s \\
& -\frac{1}{2} \int_{I \times J} \int \chi_{(t, x)}(s, y) f(y, u(s, y)) \mathrm{d} y \mathrm{~d} s \\
= & \frac{1}{2} \int_{I \times J} \int \chi_{(t, x)}(s, y)\left(f_{n}\left(y, u_{n}(s, y)\right)-f(y, u(s, y))\right) \mathrm{d} y \mathrm{~d} s \\
= & \frac{1}{2} \int_{I \times J} \int \chi_{(t, x)}(s, y)\left(f_{n}\left(y, u_{n}(s, y)\right)-f\left(y, u_{n}(s, y)\right)\right) \mathrm{d} y \mathrm{~d} s \\
& +\frac{1}{2} \int_{I \times J} \int \chi_{(t, x)}(s, y)\left(f\left(y, u_{n}(s, y)\right)-f(y, u(s, y))\right) \mathrm{d} y \mathrm{~d} s .
\end{aligned}
$$

This implies that

$$
\begin{aligned}
\left|u_{n}(t, x)-u(t, x)\right| \leq & \frac{1}{2} \int_{I \times J} \int\left|f_{n}\left(y, u_{n}(s, y)\right)-f\left(y, u_{n}(s, y)\right)\right| \mathrm{d} y \mathrm{~d} s \\
& +\frac{1}{2} \int_{I \times J} \int\left|f\left(y, u_{n}(s, y)\right)-f(y, u(s, y))\right| \mathrm{d} y \mathrm{~d} s .
\end{aligned}
$$

We define

$$
g(x)=\sup _{(s, y) \in I \times[0, x]} \mathrm{E}\left|u_{n}(s, y)-u(s, y)\right|
$$

Then, for each $x \in J$,

$$
\begin{aligned}
g(x) \leq & \frac{1}{2} \int_{0}^{T} \int_{0}^{L} \sup _{x, u}\left|f_{n}(x, u)-f(x, u)\right| \mathrm{d} y \mathrm{~d} s \\
& +\frac{1}{2} \int_{0}^{T} \int_{0}^{L} \mathrm{E}\left|f\left(y, u_{n}(s, y)\right)-f(y, u(s, y))\right| \mathrm{d} y \mathrm{~d} s \\
\leq & \frac{L T}{2} \sup _{x, u}\left|f_{n}(x, u)-f(x, u)\right|+\frac{k}{2} \int_{0}^{T} \int_{0}^{x} g(y) \mathrm{d} y \mathrm{~d} s \\
\leq & \frac{L T}{2} \sup _{x, u}\left|f_{n}(x, u)-f(x, u)\right|+\frac{k T}{2} \int_{0}^{x} g(y) \mathrm{d} y,
\end{aligned}
$$

using the Lipschitz condition and the definition of $g$. 
It follows from Gronwall's inequality (with $b=k T / 2, t_{0}=0$, and $t=x$ ) that

$$
g(x) \leq \frac{L T}{2} \mathrm{e}^{k T L / 2} \sup _{x, u}\left|f_{n}(x, u)-f(x, u)\right| .
$$

This gives (2.7), which completes the proof.

In the following result, the conclusion of Lemma 2.1 is improved and shown to hold in the $L^{p}$-sense.

Theorem 2.1. Assume that $f_{n}(x, u) \rightarrow f(x, u)$ uniformly on $J \times \mathbb{R}$. Then, for any $p \geq 2$, we obtain

$$
\sup _{(t, x) \in I \times J} \mathrm{E}\left[\left|u_{n}(t, x)-u(t, x)\right|^{p}\right] \rightarrow 0 \quad \text { as } n \rightarrow \infty .
$$

Proof. From (2.8), since $(a+b)^{p} \leq 2^{p}\left(a^{p}+b^{p}\right)$, for $0<p<\infty$ and $a, b \geq 0$, we see that

$$
\begin{aligned}
\mathrm{E}\left|u_{n}(t, x)-u(t, x)\right|^{p} \leq & \mathrm{E}\left(\int_{I \times J} \int\left|f_{n}\left(y, u_{n}(s, y)\right)-f\left(y, u_{n}(s, y)\right)\right| \mathrm{d} y \mathrm{~d} s\right)^{p} \\
& +\mathrm{E}\left(\int_{I \times J} \int\left|f\left(y, u_{n}(s, y)\right)-f(y, u(s, y))\right| \mathrm{d} y \mathrm{~d} s\right)^{p} \\
\leq & c_{p} \mathrm{E}\left(\int_{I \times J} \int\left|f_{n}\left(y, u_{n}(s, y)\right)-f\left(y, u_{n}(s, y)\right)\right|^{2} \mathrm{~d} y \mathrm{~d} s\right)^{p / 2} \\
& +c_{p} \mathrm{E}\left(\int_{I \times J} \int\left|f\left(y, u_{n}(s, y)\right)-f(y, u(s, y))\right|^{2} \mathrm{~d} y \mathrm{~d} s\right)^{p / 2} \\
\leq & c_{p}(L T)^{p} \sup _{x, u}\left|f_{n}(x, u)-f(x, u)\right|^{p} \\
& +c_{p} \mathrm{E}\left(\int_{I \times J} \int k^{2}\left|u_{n}(s, y)-u(s, y)\right|^{2} \mathrm{~d} y \mathrm{~d} s\right)^{p / 2} \\
\leq & c_{p}(L T)^{p} \sup _{x, u}\left|f_{n}(x, u)-f(x, u)\right|^{p} \text { using the Lipschitz condition } \\
& +c_{p} k^{p} \mathrm{E}\left(\int_{I \times J} \int\left|u_{n}(s, y)-u(s, y)\right|^{2} \mathrm{~d} y \mathrm{~d} s\right)^{p / 2} .
\end{aligned}
$$

Applying Hölder's inequality with indices $p / 2$ and $p /(p-2)$, we obtain

$$
\begin{aligned}
\mathrm{E}\left|u_{n}(t, x)-u(t, x)\right|^{p} & \\
\leq & c_{p}(L T)^{p} \sup _{x, u}\left|f_{n}(x, u)-f(x, u)\right|^{p} \\
& \quad+c_{p} k^{p} \mathrm{E}\left(\int_{I \times J} \int\left|u_{n}(s, y)-u(s, y)\right|^{p} \mathrm{~d} y \mathrm{~d} s\right)\left(\int_{I \times J} \int 1 \mathrm{~d} y \mathrm{~d} s\right)^{((p-2) / p) p / 2} \\
\leq & c_{p}(L T)^{p} \sup _{x, u}\left|f_{n}(x, u)-f(x, u)\right|^{p} \\
& +c_{p} k^{p}(L T)^{(p-2) / 2}\left(\int_{I \times J} \int \mathrm{E}\left|u_{n}(s, y)-u(s, y)\right|^{p} \mathrm{~d} y \mathrm{~d} s\right) .
\end{aligned}
$$


We define

$$
G(x):=\sup _{(s, y) \in I \times[0, x]} \mathrm{E}\left[\left|u_{n}(t, x)-u(t, x)\right|^{p}\right] .
$$

Then, for each $x \in J$,

$$
\begin{aligned}
G(x) & \leq c_{p}(L T)^{p} \sup _{x, u}\left|f_{n}(x, u)-f(x, u)\right|^{p}+c_{p} k^{p}(L T)^{(p-2) / 2} \int_{0}^{T} \int_{0}^{x} G(y) \mathrm{d} y \mathrm{~d} s \\
& \leq c_{1} \sup _{x, u}\left|f_{n}(x, u)-f(x, u)\right|^{p}+c_{2} \int_{0}^{x} G(y) \mathrm{d} y,
\end{aligned}
$$

where $c_{1}=c_{p} L^{p} T^{p}$ and $c_{2}=c_{p} k^{p} L^{p / 2-1} T^{p / 2}$. It follows from Gronwall's inequality that

$$
G(x) \leq c_{1} \exp \left(c_{2} L\right) \sup _{x, u}\left|f_{n}(x, u)-f(x, u)\right|^{p},
$$

and the result for $p>2$ follows. The case $p=2$ follows without using Hölder's inequality; this completes the proof.

Next, the dependence of the solutions to (2.5) on the initial condition is investigated. We first note that the process

$$
W(t):=\frac{1}{2} \int_{x-t}^{x+t} \bar{W}(\mathrm{~d} y)=\frac{1}{2} \int_{x-t}^{x+t} w(\mathrm{~d} y), \quad \text { for each } x \in J,
$$

is equivalent in distribution to a Brownian motion with variance $t / 2$. Thus, as long as the initial condition $a(x)$ is a continuous function on $J$ and $f$ is continuous in $(x, u)$, the solution $u$ to (2.1) will be in the space of continuous functions on $I \times J$. Let $C(I \times J)$ denote the vector space of continuous functions $F: I \times J \rightarrow \mathbb{R}$. Let $\|\cdot\|$ denote the uniform norm in $C(I \times J)$ or $C(J)$. The random perturbations influence the initial velocity of the amplitude of the wave

$$
\left.\frac{\partial u(t, x)}{\partial t}\right|_{t=0}
$$

while the initial displacement of the wave $u(0, x)$ is kept to a fixed continuous function $a(x)$. Hence, in the next result, $u(0, x)$ is assumed to be fixed, while

$$
\left.\frac{\partial u(t, x)}{\partial t}\right|_{t=0}
$$

varies but is still white noise in nature. In such a case, the mapping $w \rightarrow u$ is shown to be continuous.

Lemma 2.2. For each $\omega \in \Omega$, the mapping $W \rightarrow u$, defined by (2.2) and (2.3), is a continuous mapping from $C(J)$ to $C(I \times J)$ with respect to the $\|\cdot\|$-norm in these spaces. That is,

$$
\left\|u_{1}-u_{2}\right\| \leq \mathrm{e}^{k T L / 2}\left\|W_{1}-W_{2}\right\| .
$$

Proof. From (2.6) we see that

$$
\begin{aligned}
u_{1}(s, y)-u_{2}(s, y)= & \frac{1}{2} \int_{x-t}^{x+t} \bar{W}_{1}(\mathrm{~d} y)-\frac{1}{2} \int_{x-t}^{x+t} \bar{W}_{2}(\mathrm{~d} y) \\
& +\frac{1}{2} \int_{I \times J} \int \chi_{(t, x)}(s, y)\left(f\left(y, u_{1}(s, y)\right)-f\left(y, u_{2}(s, y)\right)\right) \mathrm{d} y \mathrm{~d} s .
\end{aligned}
$$


We define

$$
\mu(x):=\sup _{(s, y) \in I \times[0, x]}\left|u_{1}(s, y)-u_{2}(s, y)\right| .
$$

Then, for each $x \in J$, we see that

$$
\begin{aligned}
\mu(x) & \leq \frac{1}{2}\left\|\int_{x-t}^{x+t} \bar{W}_{1}(\mathrm{~d} y)-\int_{x-t}^{x+t} \bar{W}_{2}(\mathrm{~d} y)\right\|+\frac{k}{2} \int_{0}^{T} \int_{0}^{x} \mu(y) \mathrm{d} y \mathrm{~d} s \\
& \leq\left\|W_{1}(x)-W_{2}(x)\right\|+\frac{k T}{2} \int_{0}^{x} \mu(y) \mathrm{d} y .
\end{aligned}
$$

Applying Gronwall's inequality, we obtain

$$
\mu(x) \leq \mathrm{e}^{k T L / 2}\left\|W_{1}(x)-W_{2}(x)\right\|,
$$

and the result follows.

\section{Nonlinear random forcing}

In this section, the case of a vibrating string excited by a nonlinear stochastic force which involves the formal derivative of a Brownian sheet is considered. A Brownian sheet $W$ on $I \times J$ is a random set function defined on the Borel subsets of $I \times J$ such that the following conditions hold.

(i) For a Borel set $A$ of $B(I \times J), W(A)$ is a mean zero Gaussian random variable with covariance given by $\mathrm{E}[W(A) W(B)]=v(A \cap B)$, where $v$ is Lebesgue measure on $(I \times J, B(I \times J))$ and $B(I \times J)$ denotes the collection of Borel subsets of $I \times J$.

(ii) If $A$ and $B$ are disjoint Borel subsets of $I \times J$, then $W(A \cup B)=W(A)+W(B)$.

The Brownian sheet $W$ is defined on a fixed complete probability space $(\Omega, F, \mathrm{P})$. The filtration $\left\{F_{t}: t \geq 0\right\}$ is defined by the $\sigma$-field

$$
F_{t}:=\sigma\{W(A): A \in B([0, t] \times J)\}, \quad t \in I .
$$

The displacement $u(t, x)$ of a point $x$ at time $t$ on the string of length $L$ fixed at both ends with a Dirichlet boundary condition is given by the following version of the stochastic wave equation:

$$
\begin{aligned}
\frac{\partial^{2} u(t, x)}{\partial t^{2}} & =\frac{\partial^{2} u(t, x)}{\partial x^{2}}+f(x, u) \ddot{W}_{t x}, \\
u(0, x) & =a(x), \\
\left.\frac{\partial u(t, x)}{\partial t}\right|_{t=0} & =b(x), \\
u(t, 0) & =\left.\frac{\partial u(t, x)}{\partial t}\right|_{x=L}=0, \quad(t, x) \in I \times J,
\end{aligned}
$$

where $\ddot{W}_{t x}$ is the derivative of the Brownian sheet $W$ in the sense of distribution. The initial conditions $a(x)$ and $b(x)$ are deterministic continuous functions which are in $L^{2}(J)$. Again, we assume that $f$ is continuous as a function in $(x, u)$ on $J \times \mathbb{R}$ and Lipschitz in $u$, as in (2.4). Because of the singularity of the noise $\ddot{W}_{t x}$, a solution to (3.1) is meant in the distributional sense; see Walsh (1986) and Elshamy (1995). That is, a function $u(t, x)$ is a solution to (3.1) 
if $u(t, x)$ is a $F_{t}$-adapted continuous random field such that, for each $C^{\infty}$ function $\phi$ on $I \times J$ having compact support with $\phi(T, x)=\partial \phi(T, x) / \partial t=0$, for all $x \in J$, we have

$$
\begin{aligned}
\int_{I \times J} & \int \frac{\partial^{2} \phi(t, x)}{\partial t^{2}} u(t, x) \mathrm{d} x \mathrm{~d} t \\
= & \int_{I \times J} \int \frac{\partial^{2} \phi(t, x)}{\partial x^{2}} u(t, x) \mathrm{d} x \mathrm{~d} t-\int_{J} \frac{\partial \phi(0, x)}{\partial t} a(x) \mathrm{d} x+\int_{J} \phi(0, x) b(x) \mathrm{d} x \\
& +\int_{I \times J} \int \phi(t, x) f(x, u(t, x)) W(\mathrm{~d} x, \mathrm{~d} t), \quad \text { P-almost surely. }
\end{aligned}
$$

The solution $u(t, x)$ can then be written as

$$
u(t, x)=u^{0}(t, x)+\frac{1}{2} \int_{I \times J} \int \chi_{(t, x)}(s, y) f(y, u(s, y)) W(\mathrm{~d} y, \mathrm{~d} s), \quad(t, x) \in I \times J,
$$

where

$$
u^{0}(t, x)=\frac{1}{2}(A(x+t)+A(x-t))+\frac{1}{2} \int_{x-t}^{x+t} \bar{b}(y) \mathrm{d} y
$$

and

$$
\begin{aligned}
\bar{b}(x) & :=b(x), & & \text { for } 0 \leq x \leq L, \\
\bar{b}(-x) & :=-w(x), & & \text { for } 0 \leq x \leq L, \\
\bar{b}(x+2 k L) & =\bar{b}(x), & & \text { if }-L \leq x \leq L \text { and } k= \pm 1, \pm 2, \ldots
\end{aligned}
$$

Let $f_{n}(x, u)$ be a sequence of continuous functions defined on $J \times \mathbb{R}$ which are Lipschitz in $u$ and let $u_{n}(t, x)$ denote the solutions to the stochastic equation

$$
\begin{aligned}
\frac{\partial^{2} u_{n}(t, x)}{\partial t^{2}} & =\frac{\partial^{2} u_{n}(t, x)}{\partial x^{2}}+f_{n}\left(x, u_{n}\right) \ddot{W}_{t x}, \\
\left.\frac{\partial u_{n}(t, x)}{\partial t}\right|_{t=0} & =b(x), \\
u_{n}(t, 0) & =\left.\frac{\partial u_{n}(t, x)}{\partial t}\right|_{x=L}=0, \quad(t, x) \in I \times J .
\end{aligned}
$$

That is,

$$
u_{n}(t, x)=u^{0}(t, x)+\frac{1}{2} \int_{I \times J} \int \chi_{(t, x)}(s, y) f_{n}\left(y, u_{n}(s, y)\right) W(\mathrm{~d} y, \mathrm{~d} s), \quad(t, x) \in I \times J .
$$

Theorem 3.1. Assume that $f_{n}(x, u) \rightarrow f(x, u)$ uniformly on $J \times \mathbb{R}$, then we obtain

$$
\sup _{(t, x) \in I \times J} \mathrm{E}\left|u_{n}(t, x)-u(t, x)\right|^{2} \rightarrow 0 \quad \text { as } n \rightarrow \infty,
$$

where $u(t, x)$ satisfies $(3.4)$ with $f(x, u)$ in place of $f_{n}\left(x, u_{n}\right)$, i.e.

$$
u(t, x)=u^{0}(t, x)+\frac{1}{2} \int_{I \times J} \int \chi_{(t, x)}(s, y) f(y, u(s, y)) W(\mathrm{~d} y, \mathrm{~d} s) .
$$


Proof. Note that

$$
\begin{aligned}
u_{n}(t, x) & -u(t, x) \\
= & \frac{1}{2} \int_{I \times J} \int \chi_{(t, x)}(s, y) f_{n}\left(y, u_{n}(s, y)\right) W(\mathrm{~d} y, \mathrm{~d} s) \\
& -\frac{1}{2} \int_{I \times J} \int \chi_{(t, x)}(s, y) f(y, u(s, y)) W(\mathrm{~d} y, \mathrm{~d} s) \\
= & \frac{1}{2} \int_{I \times J} \int \chi_{(t, x)}(s, y)\left(f_{n}\left(y, u_{n}(s, y)\right)-f\left(y, u_{n}(s, y)\right)\right) W(\mathrm{~d} y, \mathrm{~d} s) \\
& +\frac{1}{2} \int_{I \times J} \int \chi_{(t, x)}(s, y)\left(f\left(y, u_{n}(s, y)\right)-f(y, u(s, y))\right) W(\mathrm{~d} y, \mathrm{~d} s) .
\end{aligned}
$$

Thus,

$$
\begin{aligned}
\mathrm{E} \mid u_{n}( & t, x)-\left.u(t, x)\right|^{2} \\
\leq & \frac{1}{2} \mathrm{E}\left|\int_{I \times J} \int\left(f_{n}\left(y, u_{n}(s, y)\right)-f\left(y, u_{n}(s, y)\right)\right) W(\mathrm{~d} y, \mathrm{~d} s)\right|^{2} \\
& +\frac{1}{2} \mathrm{E}\left|\int_{I \times J} \int\left(f\left(y, u_{n}(s, y)\right)-f(y, u(s, y))\right) W(\mathrm{~d} y, \mathrm{~d} s)\right|^{2} \\
\leq & \frac{1}{2} \mathrm{E} \int_{I \times J} \int\left|f_{n}\left(y, u_{n}(s, y)\right)-f\left(y, u_{n}(s, y)\right)\right|^{2} \mathrm{~d} y \mathrm{~d} s \\
& +\frac{1}{2} \mathrm{E} \int_{I \times J} \int\left|f\left(y, u_{n}(s, y)\right)-f(y, u(s, y))\right|^{2} \mathrm{~d} y \mathrm{~d} s \\
\leq & \frac{L T}{2} \sup _{y, u}\left|f_{n}(y, u)-f(y, u)\right|^{2} \mathrm{~d} y \mathrm{~d} s \\
& \left.+\frac{k^{2}}{2} \int_{I \times J} \int \mathrm{E} \mid u_{n}(s, y)\right)-\left.u(s, y)\right|^{2} \mathrm{~d} y \mathrm{~d} s .
\end{aligned}
$$

We define

$$
\delta(x)=\sup _{(s, y) \in I \times[0, x]} \mathrm{E}\left|u_{n}(s, y)-u(s, y)\right|^{2} .
$$

Then, for each $x \in J$,

$$
\delta(x) \leq \frac{L T}{2} \sup _{x, u}\left|f_{n}(x, u)-f(x, u)\right|^{2}+\frac{T k^{2}}{2} \int_{0}^{x} \delta(y) \mathrm{d} y .
$$

It follows from Gronwall's inequality that

$$
\delta(x) \leq \frac{L T}{2} \exp \left(\frac{k^{2} T L}{2}\right) \sup _{x, u}\left|f_{n}(x, u)-f(x, u)\right|^{2} .
$$

This gives (3.5), which completes the proof. 
We note that Theorem 3.1 actually holds for $p \geq 2$; this is stated in the following result.

Theorem 3.2. Assume that $f_{n}(x, u) \rightarrow f(x, u)$ uniformly on $J \times \mathbb{R}$. Then for any $p, p \geq 2$, we obtain

$$
\sup _{(t, x) \in I \times J} \mathrm{E}\left[\left|u_{n}(t, x)-u(t, x)\right|^{p}\right] \rightarrow 0 \quad \text { as } n \rightarrow \infty .
$$

Proof. We have

$$
\begin{aligned}
u_{n}(t, x) & -u(t, x) \\
= & \frac{1}{2} \int_{I \times J} \int \chi_{(t, x)}(s, y) f_{n}\left(y, u_{n}(s, y)\right) W(\mathrm{~d} y, \mathrm{~d} s) \\
& -\frac{1}{2} \int_{I \times J} \int \chi_{(t, x)}(s, y) f(y, u(s, y)) W(\mathrm{~d} y, \mathrm{~d} s) \\
= & \frac{1}{2} \int_{I \times J} \int \chi_{(t, x)}(s, y)\left(f_{n}\left(y, u_{n}(s, y)\right)-f\left(y, u_{n}(s, y)\right)\right) W(\mathrm{~d} y, \mathrm{~d} s) \\
& +\frac{1}{2} \int_{I \times J} \int \chi_{(t, x)}(s, y)\left(f\left(y, u_{n}(s, y)\right)-f(y, u(s, y))\right) W(\mathrm{~d} y, \mathrm{~d} s)
\end{aligned}
$$

and

$$
\begin{aligned}
& \mathrm{E}\left|u_{n}(t, x)-u(t, x)\right|^{p} \\
& \leq \mathrm{E}\left|\int_{I \times J} \int\left(f_{n}\left(y, u_{n}(s, y)\right)-f\left(y, u_{n}(s, y)\right)\right) W(\mathrm{~d} y, \mathrm{~d} s)\right|^{p} \\
& \quad+\mathrm{E}\left|\int_{I \times J} \int\left(f\left(y, u_{n}(s, y)\right)-f(y, u(s, y))\right) W(\mathrm{~d} y, \mathrm{~d} s)\right|^{p} .
\end{aligned}
$$

Using Burkholder, Hölder, and Gronwall inequalities, as in the proof of Theorem 2.1, the result follows.

Using Chebyshev's inequality and Theorem 3.1, we obtain the following result.

Lemma 3.1. Assume that $f_{n}(x, u) \rightarrow f(x, u)$ uniformly on $J \times \mathbb{R}$. Then, for any positive constant $\varepsilon$, we obtain

$$
\mathrm{P}\left(\left|u_{n}(t, x)-u(t, x)\right|>\varepsilon\right) \rightarrow 0 \text { as } n \rightarrow \infty
$$

\section{Smoothness of the random fields}

This section is devoted to the investigation of additional characteristics of the random fields representing the solutions to the stochastic wave equations (2.1) and (3.1). The integral equations (2.2) and (3.2) define a Picard iterative scheme which can be used to construct $F_{t}$-predictable elements of $L^{2}(I \times J \times \Omega)$. Standard techniques can then be utilized to show the existence of a unique $F_{t}$-predictable solution $u(t, x)$ in $L^{2}(I \times J \times \Omega)$ having a continuous version as a function of $(t, x)$. In fact, we can estimate its modulus of continuity. To this end, a function $F$ defined on a bounded domain $D$ is said to be (uniformly) Hölder continuous with 
exponent $\alpha, 0<\alpha \leq 1$, in $D$, provided that

$$
\sup \left\{\frac{|F(x)-F(y)|}{|x-y|^{\alpha}}: x, y \in D, x \neq y\right\} \text { is finite. }
$$

Hölder continuity is a quantitative measure of continuity which is particularly well suited to the study of partial differential equations. It may be viewed, in some sense, as fractional differentiability.

Theorem 4.1. (Initial white noise excitation.) Consider (2.2), the solution to Equation (2.1), where (2.3) holds.

Let $a(x)$ be a Hölder continuous function with exponent $\alpha=\frac{1}{2}$ and $f$ be a continuous function of $(x, u)$ in $J \times \mathbb{R}$. Then, for $\omega$ almost everywhere, $(x, t) \rightarrow u(x, t)$ is a Hölder continuous function with exponent $\alpha=\frac{1}{2}$.

Proof. Since $f(\cdot, \cdot)$ is a continuous function, the integral in (2.2) is a smooth function of $(t, x)$. We now estimate the increments of

$$
U(t, x)=\int_{x-t}^{x+t} \bar{W}(\mathrm{~d} y)=\int_{x-t}^{x+t} w(\mathrm{~d} y) .
$$

We have

$$
\begin{aligned}
\mathrm{E}|U(t+h, x+k)-U(t, x)| \\
\quad=\mathrm{E}|U(t+h, x+k)-U(t, x+k)+U(t, x+k)-U(t, x)| \\
\quad \leq \mathrm{E}|U(t+h, x+k)-U(t, x+k)|+\mathrm{E}|U(t, x+k)-U(t, x)| \\
\quad=\text { Term } 1+\text { Term } 2,
\end{aligned}
$$

where

$$
\begin{aligned}
\text { Term } 1 & =\mathrm{E}|U(t+h, x+k)-U(t, x+k)| \\
& =\mathrm{E}\left|\int_{x+k-t-h}^{x+k+t+h} w(\mathrm{~d} y)-\int_{x+k-t}^{x+k+t} w(\mathrm{~d} y)\right| \\
& =\mathrm{E}\left|\left(\int_{x+k-t-h}^{x+k-t}+\int_{x+k-t}^{x+k+t}+\int_{x+k+t}^{x+k+t+h}-\int_{x+k-t}^{x+k+t}\right) w(\mathrm{~d} y)\right| \\
& =\mathrm{E}\left|\int_{x+k-t-h}^{x+k-t} w(\mathrm{~d} y)+\int_{x+k+t}^{x+k+t+h} w(\mathrm{~d} y)\right| \\
& \leq \mathrm{E}\left|\int_{x+k-t-h}^{x+k-t} w(\mathrm{~d} y)\right|+\mathrm{E}\left|\int_{x+k+t}^{x+k+t+h} w(\mathrm{~d} y)\right| \\
& \leq\left(\mathrm{E}\left|\int_{x+k-t-h}^{x+k-t} w(\mathrm{~d} y)\right|^{2}+\left(\mathrm{E}\left|\int_{x+k+t}^{x+k+t+h} w(\mathrm{~d} y)\right|^{2}\right)^{1 / 2}\right. \\
& =\left(\int_{x+k-t-h}^{x+k-t} 1^{2} \mathrm{~d} y\right)^{1 / 2}+\left(\int_{x+k+t}^{x+k+t+h} 1^{2} \mathrm{~d} y\right)^{1 / 2} \\
& \leq|h|^{1 / 2}+|h|^{1 / 2} \\
& =2|h|^{1 / 2}
\end{aligned}
$$


and

$$
\begin{aligned}
\text { Term } 2 & =\mathrm{E}|U(t, x+k)-U(t, x)| \\
& =\mathrm{E}\left|\int_{x+k-t}^{x+k+t} w(\mathrm{~d} y)-\int_{x-t}^{x+t} w(\mathrm{~d} y)\right| \\
& =\mathrm{E}\left|\left(\int_{x+k-t}^{x+t}+\int_{x+t}^{x+k+t}-\int_{x-t}^{x+k-t}-\int_{x+k-t}^{x+t}\right) w(\mathrm{~d} y)\right| \\
& =\mathrm{E}\left|\int_{x+t}^{x+k+t} w(\mathrm{~d} y)-\int_{x-t}^{x+k-t} w(\mathrm{~d} y)\right| \\
& \leq \mathrm{E}\left|\int_{x+t}^{x+k+t} w(\mathrm{~d} y)\right|+\mathrm{E}\left|\int_{x-t}^{x+k-t}(-1) w(\mathrm{~d} y)\right| \\
& \leq\left(\mathrm{E}\left|\int_{x+t}^{x+k+t} w(\mathrm{~d} y)\right|^{2}+\left(\mathrm{E}\left|\int_{x-t}^{1 / 2}(-1) w(\mathrm{~d} y)\right|^{2}\right)^{1 / 2}\right. \\
& =\left(\int_{x+t}^{x+k+t} 1^{2} \mathrm{~d} y\right)^{1 / 2}+\left(\int_{x-t}^{x+k-t}(-1)^{2} \mathrm{~d} y\right)^{1 / 2} \mathrm{using} \text { Liapounov's inequality } \\
& \leq|k|^{1 / 2}+|k|^{1 / 2} \\
& =2|k|^{1 / 2} .
\end{aligned}
$$

Putting these estimates together in (4.1) and using $a+b \leq 2\left(a^{p}+b^{p}\right)^{1 / p}$, for $p \geq 1$ and $a, b \geq 0$, we see (using $p=4$ ) that

$$
\begin{aligned}
\mathrm{E}|U(t+h, x+k)-U(t, x)| & \leq 2\left(|h|^{1 / 2}+|k|^{1 / 2}\right) \\
& \leq 4\left(|h|^{2}+|k|^{2}\right)^{1 / 4} \\
& \leq 4\left(\sqrt{|t|^{2}+|k|^{2}}\right)^{1 / 2}
\end{aligned}
$$

Therefore, $U(t, x)$ is a Hölder continuous with exponent $\alpha=\frac{1}{2}$, which completes the proof.

Theorem 4.2. (Nonlinear random forcing.) Consider (3.2), the solution to Equation (3.1), where (3.3) holds.

Let $a(x)$ be a Hölder continuous function with exponent $\alpha, 0<\alpha<\frac{1}{2}$, and let

$$
\int_{I \times J} \int \mathrm{E}|f(y, u(s, y))|^{p} \mathrm{~d} y \mathrm{~d} s<\infty, \quad \text { for } p \geq 2 .
$$

Then, for $\omega$ almost everywhere, $(x, t) \rightarrow u(x, t)$ is a Hölder continuous function with exponent $\alpha, 0<\alpha<\frac{1}{2}$.

Proof. Since the function $b(\cdot)$ is continuous on $J$, the integral in (3.3) is a smooth function of $(t, x)$.

We define

$$
V(t, x)=\int_{I \times J} \int \chi_{(t, x)}(s, y) f(y, u(s, y)) W(\mathrm{~d} y, \mathrm{~d} s), \quad(t, x) \in I \times J .
$$


We show that, for $\omega$ almost everywhere, $V(t, x)$ is a Hölder continuous function by estimating its increments. We have

$$
\begin{aligned}
(\mathrm{E} \mid V & \left.(t+h, x+k)-\left.V(t, x)\right|^{n}\right)^{1 / n} \\
& =\left(\mathrm{E}|V(t+h, x+k)-V(t, x+k)+V(t, x+k)-V(t, x)|^{n}\right)^{1 / n} \\
& \leq\left(\mathrm{E}|V(t+h, x+k)-V(t, x+k)|^{n}\right)^{1 / n}+\left(\mathrm{E}|V(t, x+k)-V(t, x)|^{n}\right)^{1 / n} \\
& \quad \text { by Minkowski’s inequality } \\
& =\text { Term A + Term B, }
\end{aligned}
$$

where

$$
\begin{aligned}
(\text { Term A })^{n}= & \mathrm{E}|V(t+h, x+k)-V(t, x+k)|^{n} \\
= & \mathrm{E} \mid \int_{I \times J} \int \chi_{(t+h, x+k)}(s, y) f(y, u(s, y)) W(\mathrm{~d} y, \mathrm{~d} s) \\
& -\left.\int_{I \times J} \int \chi_{(t, x+k)}(s, y) f(y, u(s, y)) W(\mathrm{~d} y, \mathrm{~d} s)\right|^{n} \\
= & \mathrm{E}\left|\int_{I \times J} \int\left(\chi_{(t+h, x+k)}(s, y)-\chi_{(t, x+k)}(s, y)\right) f(y, u(s, y)) W(\mathrm{~d} y, \mathrm{~d} s)\right|^{n} \\
\leq & \left.c \mathrm{E} \mid \int_{I \times J} \int\left(\chi_{(t+h, x+k)}(s, y)-\chi_{(t, x+k)}(s, y)\right)^{2} f^{2}(y, u(s, y)) \mathrm{d} y \mathrm{~d} s\right)\left.\right|^{n / 2} \\
\leq & c\left(\int_{I \times J} \int\left|\chi_{(t+h, x+k)}(s, y)-\chi_{(t, x+k)}(s, y)\right|^{2 q} \mathrm{~d} y \mathrm{~d} s\right)^{n / 2 q} \\
& \times \mathrm{E}\left(\int_{I \times J} \int\left|f^{2}(y, u(s, y))\right|^{p} \mathrm{~d} y \mathrm{~d} s\right)^{n / 2 p} \\
\leq & c\left(\int_{I \times J} \int\left|\chi_{(t+h, x+k)}(s, y)-\chi_{(t, x+k)}(s, y)\right|^{2 q} \mathrm{~d} y \mathrm{~d} s\right)^{n / 2-1} \\
& \times \int_{I \times J} \int \mathrm{E}|f(y, u(s, y))|^{n} \mathrm{~d} y \mathrm{~d} s .
\end{aligned}
$$

Here, (4.3) is obtained by Burkholder's inequality, where $c$ is a constant whose value may change from line to line in this proof, and (4.4) is obtained using Hölder's inequality with $p=n / 2$ and $q=n /(n-2)$.

We observe that $\chi_{(t+h, x+k)}(s, y)-\chi_{(t, x+k)}(s, y)$ vanishes except in the shaded region between two rectangles with long sides making $45^{\circ}$ with the $x$-axis (see Figure 2), where its value is either +1 or -1 . Therefore, the first integral can be estimated by maximizing the shaded area, which is easily seen to be no larger than

$$
4(\sqrt{2} L)\left(\frac{h}{\sqrt{2}}\right)=4 L h .
$$

Therefore,

$$
\begin{aligned}
\text { Term } \mathrm{A} & \leq c(4 L h)^{1 / 2-1 / n}\left(\int_{I \times J} \int \mathrm{E}|f(y, u(s, y))|^{n} \mathrm{~d} y \mathrm{~d} s\right)^{1 / n} \\
& \leq c(4 L h)^{1 / 2-1 / n} .
\end{aligned}
$$




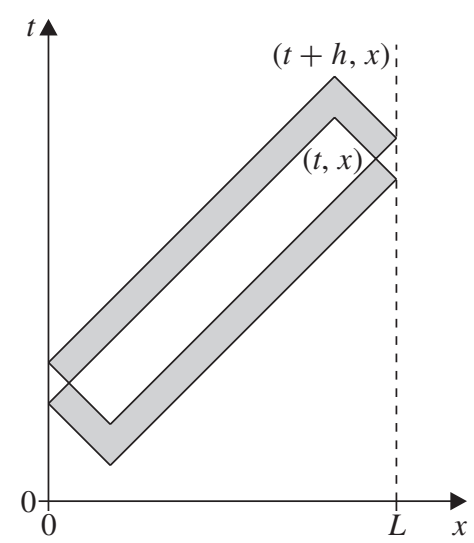

FIGURE 2: Illustration of $\chi_{(t+h, x)}(s, y)-\chi_{(t, x)}(s, y)$.

Similarly,

$$
\text { Term } \mathrm{B} \leq c(4 L k)^{1 / 2-1 / n} .
$$

Putting these bounds together in (4.2), we see that

$$
\begin{aligned}
\left(\mathrm{E}|V(t+h, x+k)-V(t, x)|^{n}\right)^{1 / n} & \leq c(4 L h)^{1 / 2-1 / n}+c(4 L h)^{1 / 2-1 / n} \\
& \leq c\left(h^{1 / 2-1 / n}+k^{1 / 2-1 / n}\right) \\
& \leq c\left(h^{(1 / 2-1 / n) p}+k^{(1 / 2-1 / n) p}\right)^{1 / p} \\
& \leq c\left(\sqrt{h^{2}+k^{2}}\right)^{1 / 2-1 / n}, \quad \text { with } p=\frac{4 n}{n-2} .
\end{aligned}
$$

Since $n$ can be chosen to be as large as we please, it follows from Liapounov's inequality that $V(t, x)$ is a Hölder continuous function with exponent $\alpha, 0<\alpha<\frac{1}{2}$. This completes the proof.

\section{Acknowledgement}

The author would like to thank an anonymous referee for making some important remarks which brought about improvements, both in form and in the content, to the original manuscript.

\section{References}

Belinskiy, B. P. AND Caithamer, P. (2001). Energy of a string driven by a two-parameter Gaussian noise white in time. J. Appl. Prob. 38, 960-974.

CABAÑA, E. M. (1972). On barrier problems for the vibrating string. Z. Wahrscheinlichkeitsth. 22, $13-24$.

Cabaña, E. M. (1991). A Gaussian process with parabolic variance. J. Appl. Prob. 28, 898-902.

Elshamy, M. (1995). Randomly perturbed vibrations. J. Appl. Prob. 32, 417-428.

Elshamy, M. (1996). Stochastic models of damped vibrations. J. Appl. Prob. 33, 1159-1168.

Orsingher, E. (1984). Damped vibrations excited by white noise. Adv. Appl. Prob. 16, 562-584.

ORsingher, E. (1989). On the maximum of Gaussian Fourier series emerging in the analysis of random vibrations. J. Appl. Prob. 26, 182-188.

Walsh, J. B. (1986). An introduction to stochastic partial differential equations. In École d'Été de Probabilitiés de Saint-Flour, XIV—1984 (Lecture Notes Math. 1180), Springer, New York, pp. 265-439. 